\title{
Elliptic and Concentric-Elliptic Antenna Array Design using Grey Wolf Optimization
}

\author{
Abdelmadjid Recioui*, Oussama Traikia and Youcef Grainat \\ Laboratory of Signals and Systems, Institute of Electrical and Electronic Engineering \\ University M'hamed Bougara of Boumerdes \\ Boumerdes, Algeria. \\ *a recioui@univ-boumerdes.dz
}

\begin{abstract}
In this work, Grey Wolf Optimization (GWO) is applied to the optimization of elliptical and concentric elliptical antenna arrays. The objective is to minimize the Sidelobe level and improve the directivity. GWO is a nature-inspired meta-heuristic algorithm inspired by the social hierarchy and hunting behavior of grey wolves. The obtained optimal values result in a good reduction of the sidelobe level for the elliptical and concentric elliptical antenna arrays with an enhancement in the directivity. This makes the designed arrays of practical use in the communication systems.
\end{abstract}

Keywords: Elliptic antenna arrays, Grey wolf optimization, optimization, directivity, sidelobe level

\section{INTRODUCTION}

Wireless communication has become vital and essential in our everyday life. An important contribution to the development of this technology is due to the advances by leaps in antenna design that made it possible to implement highly efficient and compact wireless devices. The antenna is one of the most critical components in a communication system. It is the transitional structure between the transmission line and free space through which the electromagnetic waves travel. In other words it is the system that collects and converts the radio waves into electrical signals in receiving mode. The opposite is done when the antenna is used in a transmitter system. The quality of the antenna and its characteristics will significantly affect the overall performance of a wireless communication system. For this reason, engineering has demonstrated great interest for antennas with the objective to adapt the conception to many applications by improving the characteristics of the antennas like Directivity, Sidelobe Level (SLL), or physical layout such that it suits the given application [1-2].

It was found that multiple antennas can be combined to operate together, resulting in an antenna array structure that can

Cite this article as:

A. RECIOUI, O. TRAIKIA and Y. GRAINAT, "Elliptic and Concentric-Elliptic Antenna Array Design using Grey Wolf Optimization", Algerian Journal of Signals and Systems, Vol. 4, Issue 1, June 2019.pp: 32-38. achieve a better performance, with a more flexible radiation pattern that can be tailored to meet the specifications thanks to the various geometries the array can take, and the several possible ways to feed its individual elements. Many array geometries were used in practice, from simple configurations with few elements, to more complex arrays having more elements such as planar and conformal arrays [1-2].

An important class of arrays is the elliptic array that is used in various applications including mobile communications and radar thanks to its advantageous geometry and angular symmetry [3-4]. Another version of this array is the concentric elliptic Array (CEA). It consists of elements arranged in multiple concentric ellipses that result in an antenna with very low SLL. This antenna can be optimized further using various approaches and different optimization techniques [5-7].

Optimization has been extensively used in several fields of science and engineering to solve various problems. This is due to the advent of modern global non-classical techniques that handle non-differentiable and non-linear complex functions in conjunction with the processing power of the quickly evolving computers. Various optimization techniques have been used in the field of electromagnetics. Examples include Genetic Algorithms (GA) [8-9], Differential Evolution (DE), and Particle Swarm Optimization (PSO) [10-11], that belong to the class of evolutionary techniques inspired from nature. Other recent 
techniques have also been employed in antenna design such as the Taguchi method [12-13], which is based on the concept of fractional factorial design, and makes use of Orthogonal Arrays (OAs) that significantly reduce the number of iterations needed in the optimization process. The Galaxy-based Search Algorithm (GbSA) is another natureinspired metaheuristic technique that mimics the behavior of spiral galaxies when searching its surroundings [14].

Grey wolf optimization is a swarm intelligent technique developed by Mirjalili et al [15], which emulates the grey wolves hunting technique and the social hierarchy that are mathematically modeled to design GWO and perform optimization. GWO algorithm has few parameters and easy to implement, which make it superior than gravitational search algorithm (GSA), particle swarm optimization (PSO), and fast evolutionary programming [15]. GWO has gained much attention and has been used to deal with a number of practical optimization problems such as such as optimal power flow [16], twostage assembly flow shop scheduling problem [17], unit commitment problem [18], feature selection [19], training multi-layer perceptrons [20], optimization of reactive power dispatch [21], solutions to benchmarks generally used to test optimization algorithms [22], hyperspectral band selection [23], maximum power point tracking [24], and optimal tuning of PI- and PID-fuzzy controllers [25-27]. In the literature, researchers proposed improvements of GWO. These are namely: GWO algorithm based on Powell local optimization method for global optimization and clustering analysis [28], a hybrid GWO (HGWO) algorithm with differential evolution (DE) [29], evolutionary population dynamics (EPD) in the grey wolf optimizer [30], Mean Grey Wolf Optimizer (MGWO) [31], a variant of the GWO called Modified GWO (mGWO) [32] , a modified discrete GWO variant L. Li et al. [33], an intelligent grey wolf optimizer variant called DCS-GWO [34] and finally two novel optimization techniques called Salp Swarm Algorithm (SSA) and Multi-objective Salp Swarm Algorithm (MSSA) for finding the solution of global optimization functions have been proposed in [34].

In many applications, it is desired to design an antenna with a low SLL in order minimize interference. However, reducing the sidelobes generally results in decreasing directivity, which is required to be high in order to increase power efficiency and avoid useless electromagnetic pollution. In this work, Elliptic and concentric elliptic antenna Arrays are optimized to achieve a better performance both in terms of SLL and Directivity using grey wolf Optimization (GWO). GWO is a new nature-inspired metaheuristic algorithm inspired by the social hierarchy and hunting behavior of grey wolves. It has the potential to have high performance in solving both unconstrained and constrained optimization problems [3536]. The task is performed by varying the elements' excitation amplitudes and the elliptic array eccentricity.

\section{PROBLEM FORMULATION}

\section{A. Elliptic antenna arrays (EAA)}

The geometry of an elliptical antenna array (EAA) with $\mathrm{N}$ isotropic antenna elements the $x-y$ plane is shown in Fig.1. The origin is considered to be the center of an ellipse. The array factor for this elliptical array is given by $[3,4]$ :

$$
A F(\theta, \varphi)=\sum_{n=1}^{N} I_{n} \exp \left(\left[k \sin \theta\left(a \cos \varphi_{n} \cos \varphi+b \sin \varphi_{n} \sin \varphi\right)+\alpha_{n}\right]\right)(1)
$$

Where:

$$
\begin{aligned}
& k=\frac{2 \pi}{\lambda} \\
& \varphi_{n}=\frac{2 \pi(n-1)}{N}
\end{aligned}
$$

In the above equations, $I_{n}$ and $\alpha_{n}$ represent the excitation amplitude and phase of the nth element.

$\varphi_{n}$ is the angular position of the element in the $x-y$ plane, $\varphi$ is the azimuth angle measured from the positive $x$ axis, $\theta$ is the elevation angle measured from the positive $z$ axis.

To direct the peak of the main beam in the ( $\left.\theta_{0}, \varphi_{0}\right)$ direction, the excitation phase is chosen to be:

$$
\alpha_{n}=-k \sin \theta_{0}\left(a \cos \varphi_{n} \cos \varphi_{0}+b \sin \varphi_{n} \sin \varphi_{0}\right)(4)
$$

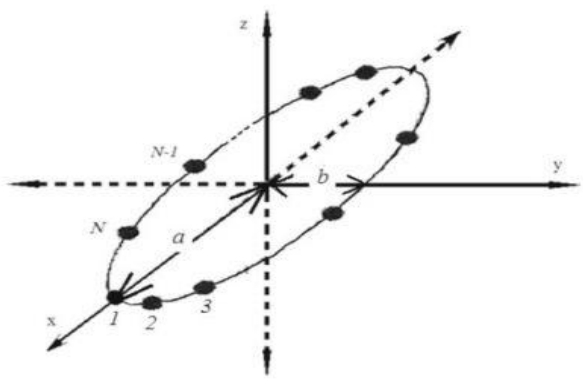

Fig. 1 Geometry of an elliptical antenna array (EAA) with isotropic radiators. 


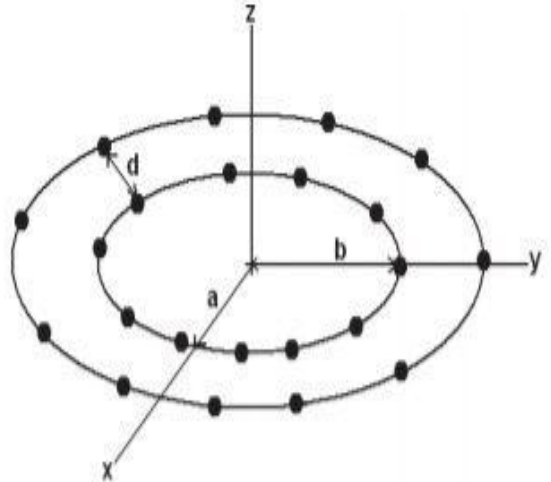

Fig. 2 Geometry of concentric elliptic array

\section{B. Concentric Elliptic Antenna Array (CEAA)}

if $\mathrm{N}$ is the number of antenna elements lie on ellipses and $M$ is the number of concentric ellipses, then the total array factor of the concentric elliptical array arrangement of isotropic elements is expressed as [3,4]:

$\left.A F(\theta, \Phi)=\sum_{m=1}^{M} \sum_{n=1}^{W} B_{\pi m} \exp \left(\left[k \sin (\theta)\left(a_{m} \cos (\phi n) \cos (\phi)+b_{\pi m} \sin (\phi n)\right) \sin (\phi)\right)\right]\right)$

$B_{m n}$ is the amplitude of excitation current, $a_{m}$ and $b_{m}$ are semi major axis and semiminor axis of $m$-th elliptical array, respectively. If "a" is the smallest semi-major axis and "d" is the spacing between ellipses in Fig. 2, then

$$
\begin{gathered}
\mathbf{a}_{\mathrm{m}}=\mathbf{a}+(\mathbf{m}-\mathbf{1}) \mathbf{d} \\
b_{m}=a_{m} \sqrt{\mathbf{1}-\mathbf{e}^{\mathbf{2}}}
\end{gathered}
$$

The eccentricity of elliptic antenna array is defined as follows:

$$
\mathrm{e}=\sqrt{1-\frac{b^{2}}{a^{2}}}
$$

Where $a, b$ are the semi-major and semiminor axis of the elliptic antenna (see Fig. 1), respectively.

The objective of the optimization is to obtain an array factor with the least possible sidelobe level (SLL) with a practically acceptable directivity. Three fitness functions are used for the three objectives: Minimize SLL and maximize Directivity separately and at last, optimize both characteristics: Mathematically, the functions are given in a respective manner as:

$$
\text { Fitness SLL= SLL }=20 \log \left(\left|\frac{A F}{\operatorname{Max}(A F)}\right|\right.
$$

$F$ itness DIR= $-\mathrm{DIR}=-(41253 / \mathrm{HPBW})(10)$

$$
F \text { itness SLL and DIR=SLL-DIR }
$$

In the equations, AF stands for the array factor and HPBW is the half power beamwidth.

\section{GREY WOLF OPTIMIZATION}

The Grey Wolf Optimizer (GWO) algorithm lies on the basis of modeling grey wolf social hierarchy and hunting habits towards finding a prey. This social hierarchy is simulated by categorizing the population of $n$ wolves is defined as the third leader in the group, which dominates the omega wolves. Mathematically, the top three fittest solutions in GWO are called alpha ( $\alpha$ ), beta ( $\beta)$, and delta $(\delta)$, respectively. The rest are assumed to be omega $(\omega)$. In GWO, the hunting process is guided by $\alpha, \beta$, and $\delta$, while $\omega$ follows these three leaders.

In GWO, the first three best solutions obtained are stored and push the other search agents to update their positions due to the position of these best search agents. In order to formulate the social hierarchy of wolves when designing GWO algorithm, the population is split into four groups: alpha ( $\alpha$ ), beta $(\beta)$, delta $(\delta)$ and omega $(\omega) . \alpha$ is linearly decreased from 2 to 0 as the optimization process evolves. The rest of the candidate solutions are denoted as $\omega$. In this algorithm, the hunting/optimization is guided by $\alpha, \beta, \delta$ and $\omega$. The wolves are required to encircle $\alpha, \beta$ and $\delta$ to find better solutions $[15,30,37-39]$.

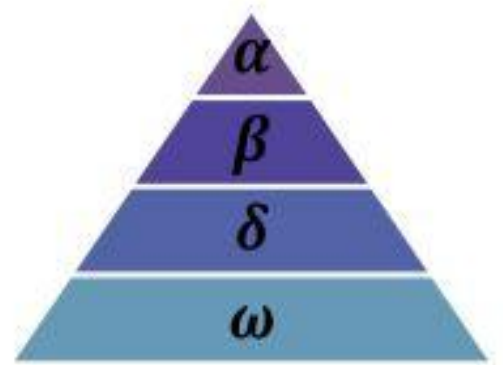

Fig. 3 Hierarchy of grey wolf (dominance decreases from top down).

\section{Mathematical modeling and algorithm}

In this section, the mathematical models of the social hierarchy, tracking, encircling, and attacking prey are provided. Then, the GWO algorithm is outlined.

Grey wolves are species with a very firm social dominant hierarchy of leadership. They live in a pack with a group size of 5 to 12 . The leaders are a male and a female, called alpha. The alpha is mostly responsible for making decisions about hunting, sleeping place, time to wake, and so on. The alphas decisions are dictated to the pack. The second level in the hierarchy of grey wolves is beta. The betas are subordinate wolves 
that help the alpha in decision-making or other pack activities. The beta wolf is the best candidate to be the alpha in case one of the alpha wolves passes away or becomes very old to lead. The lowest ranking grey wolf is omega. The omega plays the role of scapegoat. Omega wolves always have to submit to all the other dominant wolves. They are the last wolves that are allowed to eat. The fourth class is called subordinate (or delta in some references). Delta wolves have to submit to alphas and betas, but they dominate the omega. Scouts, sentinels, elders, hunters, and caretakers belong to the delta category and each has its own defined responsibilities.

In GWO algorithm, the fittest solution is called the alpha $(\alpha)$ while the second and third best solutions are named beta $(\beta)$ and delta ( $\delta)$ respectively. The rest of the candidate solutions are assumed to be omega $(\omega)$. The hunting is guided by $\alpha, \beta$, and $\delta$ and the $\omega$ follow these three candidates. The hunting behavior is mainly divided into three steps [40-41]:

a) Tracking, chasing and approaching the prey.

b) Encircling and harassing the prey until it stops moving.

c) Attacking the prey.

The encircling behavior for the pack to hunt a prey can be expressed as:

$$
X(t+1)=X_{p}(t)-A \cdot D
$$

where $X p$ is the position of prey, $A$ is the coefficient vector, and $D$ is defined as:

$$
D=\left|C \cdot X_{p}(t)-X(t)\right|
$$

where $C$ is the coefficient vector, $X$ is the position of grey wolf, and $t$ is the number of iterations. The coefficient vectors, A and C, are found as:

$$
\left\{\begin{array}{c}
A=2 a \cdot r_{1}-a \\
C=2 r_{2}
\end{array}\right.
$$

Where $r_{1}$ and $r_{2}$ are two independent random numbers uniformly distributed between [0, 1], and $a$ is the encircling coefficient that is used to balance the trade-off between exploration and exploitation. In GWO, the parameter $a$ is linearly decreased from 2 to 0 as:

$$
a=2-2\left(\frac{t}{T}\right)
$$

where $t$ is the number of iterations, and $T$ is the maximum number of iterations. In GWO, the leader alpha, beta, and delta wolves are known to have better knowledge about the potential position of prey. The leaders are guiding the omega wolves to move toward the optimal position. Mathematically, the new position of the wolf is updated as:

$$
X(t+1)=\frac{X_{1}+X_{2}+X_{3}}{3}
$$

Where $\mathrm{X}_{1}, \mathrm{X}_{2}$, and $\mathrm{X}_{3}$ are calculated as:

$$
\left\{\begin{array}{l}
X_{1}=\left|X_{\alpha}-A_{1} \cdot D_{\alpha}\right| \\
X_{2}=\left|X_{\beta}-A_{2} \cdot D_{\beta}\right| \\
X_{3}=\left|X_{\delta}-A_{3} \cdot D_{\delta}\right|
\end{array}\right.
$$

where $X_{\alpha}, X_{\beta}$, and $X_{\delta}$ are the position of alpha, beta, and delta at iteration t. $A_{1}, A_{2}$, and $\mathrm{A}_{3}$ are calculated following Equation (14); and $D_{\alpha}, D_{\beta}$ and $D_{\delta}$ are defined as:

$$
\left\{\begin{array}{l}
D_{\alpha}=\left|C_{1} \cdot X_{\alpha}-X\right| \\
D_{\beta}=\left|C_{2} \cdot X_{\beta}-X\right| \\
D_{\delta}=\left|C_{3} \cdot X_{\delta}-X\right|
\end{array}\right.
$$

where $\mathrm{C}_{1}, \mathrm{C}_{2}$, and $\mathrm{C}_{3}$ are calculated based on Equation (14).

\section{Flowchart of main GWO Algorithm}

The following figure is a flowchart that describes the simplified algorithm of the GWO.

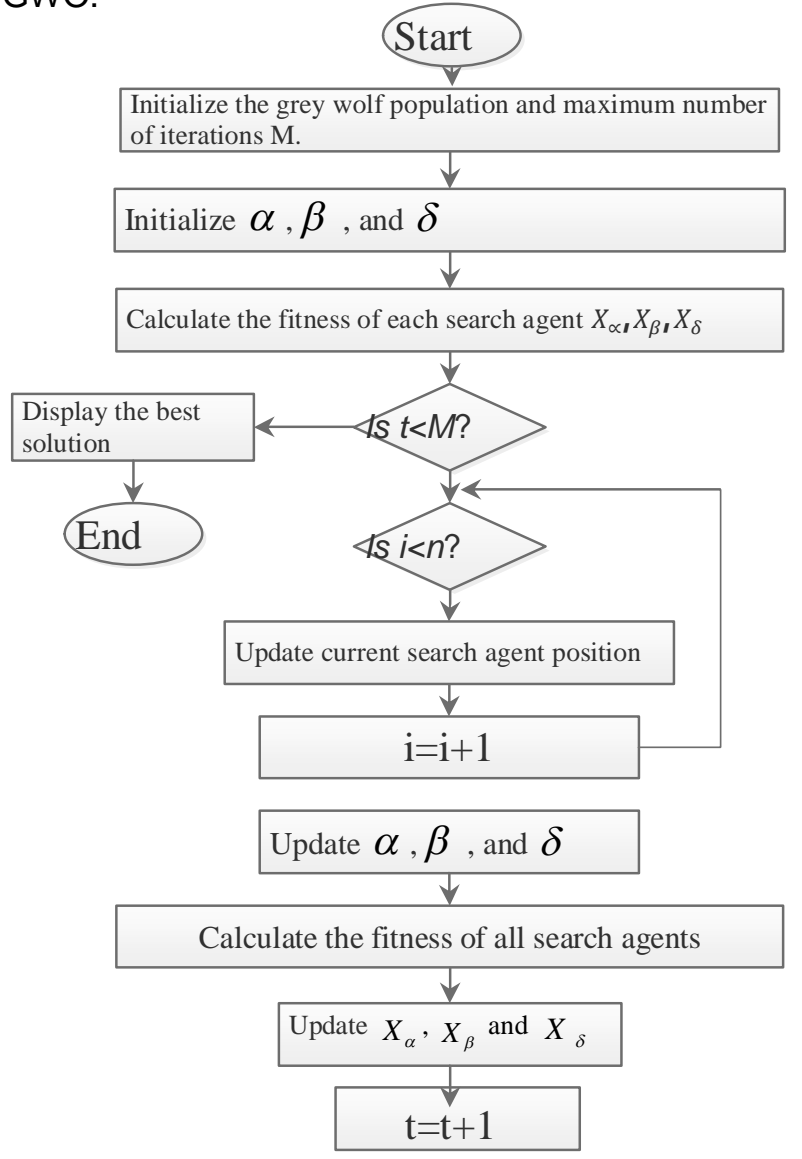

Fig. 4 Flowchart of the grey wolf optimization algorithm. 


\section{RESULTS AND DISCUSSIONS}

\section{E. Effect of eccentricity}

The eccentricity is found to affect the radiation characteristics. A parametric study is done for the case of uniform elliptic array. The results are summarized in table 1.

Table 1 radiation properties for different values of eccentricity

\begin{tabular}{|c|c|c|c|}
\hline Eccentricity & 0.51 & 0.86 & 0.93 \\
\hline SLL & $-8.08 \mathrm{~dB}$ & $-11.6 \mathrm{~dB}$ & $-16.37 \mathrm{~dB}$ \\
\hline Dir & $35.9 \mathrm{~dB}$ & $35.001 \mathrm{~dB}$ & $34.96 \mathrm{~dB}$ \\
\hline
\end{tabular}

The results in Table 1 suggest that the eccentricity must be taken into account as a parameter in the optimization process.

\section{F. Optimization of the simple elliptic array}

The fitness values in equations (9) through (11) are used in conjunction with (1) for an elliptic array having 20 elements. The results of the optimization are shown in table 2. Fig. 5 shows the resulting array factor for the case of optimizing for SLL-only.

Table 2 the result of the optimization of simple EAA.

\begin{tabular}{|l|c|c|c|}
\cline { 2 - 4 } \multicolumn{1}{c|}{} & SLL (dB) & $\begin{array}{c}\text { DIR } \\
(\mathbf{d B})\end{array}$ & Ratio $\left(\begin{array}{|c|}\mathbf{\text { DIR }} \\
\right.$\cline { 2 - 4 }\end{array}$)$ \\
\hline Uniform & -8.107 & 35.82 & 4.41 \\
\hline $\begin{array}{l}\text { Optimizing } \\
\text { SLL }\end{array}$ & -15.95 & 34.22 & 2.15 \\
\hline Optimizing DIR & -7.89 & 38.37 & 4.86 \\
\hline $\begin{array}{l}\text { Optimizing } \\
\text { both }\end{array}$ & -9.25 & 34.65 & 3.76 \\
\hline
\end{tabular}

It can be noticed that the case of optimizing for sidelobe level reduction only produces the best compromise between the sidelobe level and the directivity. This suggests that the uniform case is not the best in terms of compromise between the two characteristics as it exhibits a slightly better directivity but a worst sidelobe level. It should be noted that the sidelobe level obtained here is of practical use in the modern communication systems especially in radar applications.

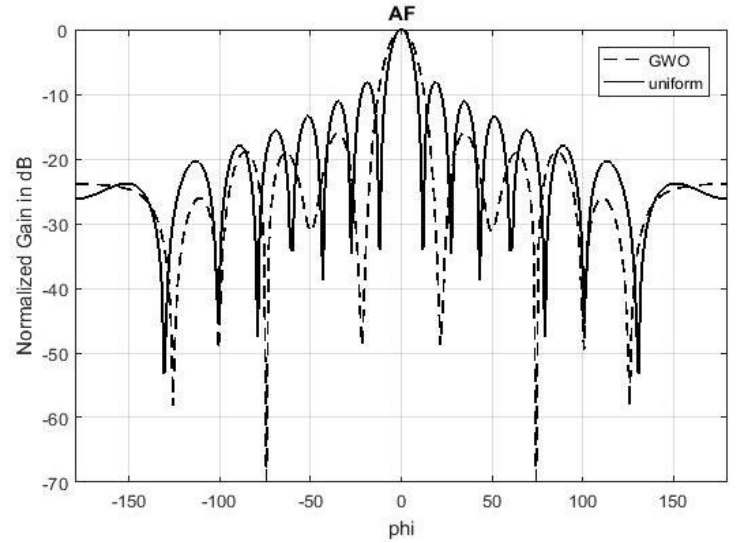

Fig.5 pattern of EAA when optimizing for SLL only

\section{G. Optimization of the concentric elliptic array}

In this section, the array factor in (5) is used along with the fitness functions in (9) through (11). The number of concentric ellipses is set to 3 . The number of elements in each ellipse is set to 20 making a total of 60 elements. The ellipses are assumed to have the same eccentricity (same ratio of $a$ and $b$ in Fig. 2). This makes the elements in the ellipses have the same values of $\varphi_{n}$. The parameters to be varied are the element excitations and the eccentricity.

Table 3 summarizes the obtained results for the three fitness values and Fig. 6 presents the array factor for the in the case of optimizing for the sidelobe only.

Again, the case of optimizing the sidelobe level produces the best compromise between directivity and sidelobe level. The value of the sidelobe level obtained fits well the practical communication applications (in radar applications, a sidelobe level of $-20 \mathrm{~dB}$ is enough for target detection). The directivity obtained in this case is not that worse compared to the uniform case.

Comparing the results in tables 2 and 3 , one would remark that the optimized sidelobe level and the optimized directivity are much better than the SLL and the directivity of uniform one for both antenna array geometries. With regards to the resulting values of the sidelobe level and directivity along with their ratios, it is clear that the concentric elliptic array produces better results compared to the simple elliptic array as more elements are involved in the optimization task. 
Table 3 the result of the optimization of CEA.

\begin{tabular}{|l|c|c|c|}
\cline { 2 - 4 } \multicolumn{1}{c|}{} & $\begin{array}{c}\text { SLL } \\
(\mathbf{d B})\end{array}$ & $\begin{array}{c}\text { DIR } \\
(\mathbf{d B})\end{array}$ & Ratio( $\left(\begin{array}{|l|}\text { (SLL) } \\
\hline \text { Uniform }\end{array}\right)$ \\
\hline OptimizingSLL & -31.56 & 33.93 & 1.075 \\
\hline OptimizingDIR & -7.89 & 38.23 & 4.86 \\
\hline Optimizingboth & -12.59 & 34.94 & 4.03 \\
\hline
\end{tabular}

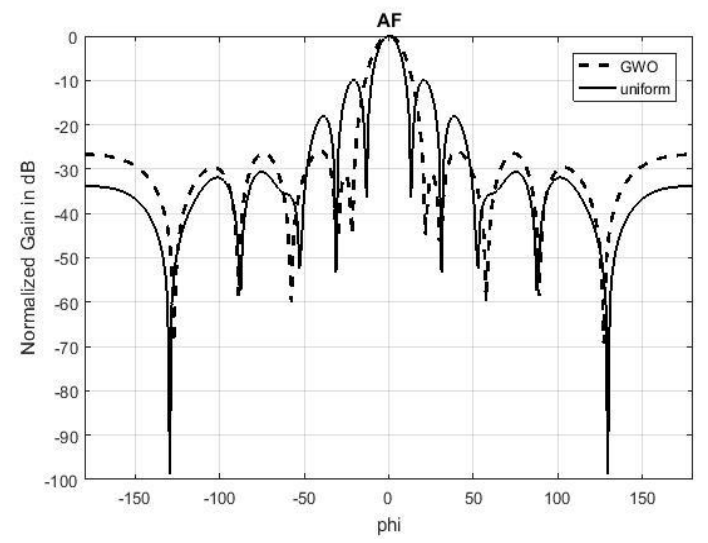

Fig. 6 pattern of CEAA when optimized for SLL

only

\section{CONCLUSIONS}

In this work, a relatively new natureinspired global optimization technique, called Grey wolf optimization (GWO) is used for the optimization of simple elliptic and concentric elliptic antenna arrays. GWO is inspired from the social hierarchy and hunting behavior of grey wolves. In the GWO algorithm, the hunting (optimization) is guided by $\alpha, \beta$, and $\delta$ factors. The remaining set of candidate solutions are denoted as omega $(\omega)$. The GWO algorithm produces the nonuniform excitation amplitudes for the elliptical and concentric elliptical arrays in question. The performance of the antennas arrays wee optimized in terms of sidelobe level and directivity. Another parameter has been found to affect the performance of the arrays is the eccentricity factor that has been embedded into the optimization problem along with the element excitations. The simulated results reveal that the optimal design offers a considerable SLL reduction at comparable Directivity relative to the conventional uniform arrays.

\section{REFERENCES}

[1] Abdelmadjid Recioui, Mondher Benabid and Nabil Djilani, "Rectangular Antenna Array Optimization using Wind Driven Optimization", Algerian Journal of Signals and Systems, Vol.1, Issue2, December 2016. pp:109-120.

[2] Abdelmadjid Recioui, "Application of the Spiral Optimization Technique to Antenna Array Design", Book chapter in: Handbook of Research on Emergent Applications of Optimization Algorithms, IGI Global, 2018.

[3] Neyestanak, A.; Ghiamy, M.; Moghaddasi, M.; Saadeghzadeh, R.: An investigation of hybrid elliptical antenna arrays. IET Microw. Antennas Propag. 2(1), 28-34 (2008)

[4] Saadeghzadeh, R.; Neyestanak, A.; Moghaddasi, M.; Ghiamy, M.: A comparison of various hybrid elliptical antenna arrays. Iran. J.Electr. Comput. Eng. 7(2), 98-106 (2008)

[5] Priyanka Das, Jibendu Sekhar Roy, "Thinning of Elliptical Antenna Arrays Using Genetic Algorithm", Int. Journal of Engineering Research and Applications, Vol. 4, issue4, April 2014, pp:225-229.

[6] Ashraf Sharaqa - Nihad Dib, "Design of Linear and Elliptical Antenna Arrays Using Biogeography Based Optimization", Arabian journal of science and engineering, 2013. DOI 10.1007/s13369-013-0794-8.

[7] Ahmidi, N.; Neyestanak, A.; Dawes, R.: Elliptical array antenna design based on particle swarm method using fuzzy decision rules. In: 24th Biennial Symposium on Communications, Kingston, ON, pp. 352-355 (2008).

[8] A. Recioui and A. Azrar (2007), "Use of Genetic Algorithms in Linear and Planar Antenna," Microwave And Optical Technology Letters, Vol. 49, No. 7.

[9] Recioui Abdelmadjid, Arab Azrar, Hamid Bentarzi, Mokrane Dehmas \& Mouloud Challal (2008). Synthesis of Linear Arrays with Sidelobe Level Reduction Constraint using Genetic Algorithms, international journal of microwave and optical technology, Vol. 3, No. 5.

[10] Khodier, M. M. and C. G. Christodoulou, Linear array geometry synthesis with minimum side lobe level and null control using particle swarm optimization," IEEE Trans. on Antennas Propagate, Vol. 53, No. 8, 26742679, August 2005.

[11] Recioui A ., Sidelobe Level Reduction in Linear Array Pattern Synthesis Using Particle Swarm Optimization", J. of Optimization Theory and Applications 153(2), 2012, pp: 497-512. DOI 10.1007/s10957-011-9953-9.

[12] Dib N., Goudos S. and Muhsen H. (2010), Application of taguchi's optimization method and self-adaptive differential evolution to the 
synthesis of linear antenna arrays. PIER, vol. $102: 159-180$.

[13] Recioui Abdelmadjid (2014), Optimization of Antenna Arrays Using Different Strategies Based on Taguchi Method, Arabian Journal for Science and Engineering 39(2) : 935-944.

[14] Abdelmadjid Recioui, Application of a GalaxyBased Search Algorithm to MIMO System Capacity Optimization, Arabian Journal for Science and Engineering, (2015), DOI: 10.1007/s13369-015-1934-0

[15] Seyedali Mirjalili, Seyed Mohammad Mirjalili, Andrew Lewis. Grey Wolf Optimizer. Advances in Engineering Software; 2014; 69: 46-61.

[16] A. A. El-Fergany, H. M. Hasanien: Elec. Power Compon. Syst. 43 (2015), p. 1548

[17] G. M. Komaki, V. Kayvanfar: J. Comput. Sci. 8 (2015), p. 109

[18] V. K. Kamboj: Neural Comput. Applic. 27 (2016), p. 1643

[19] E. Emary, H. M. Zawbaa and A. E. Hassanien: Neuroomput. 172 (2016), p. 371.

[20] Mirjalili, S. How effective is the grey wolf optimizer in training multi-layer perceptrons. Appl. Intell. 2015, 43, 150-161.

[21] Sulaiman, M.H.; Mustaffa, Z.; Mohamed, M.R.; Aliman, O. Using the grey wolf optimizer for solving optimal reactive power dispatch problem. Appl. Soft Comput. 2015, 32, 286-292.

[22] Saremi, S.; Mirjalili, S.Z.; Mirjalili, S.M. Evolutionary population dynamics and grey wolf optimizer. Neural Comput. Appl. 2015, 26, 1257-1263.

[23] Medjahed, S.A.; Saadi, T.A.; Benyettou, A.; Ouali, M. Gray wolf optimizer for hyperspectral band selection. Appl. Soft Comput. 2016, 40, 178-186.

[24] Yang, B.; Zhang, X.-S.; Yu, T.; Shu, H.-C.; Fang, Z.-H. Grouped grey wolf optimizer for maximum power point tracking of doubly-fed induction generator based wind turbine. Energy Convers. Manag. 2017, 133, 427443.

[25] Noshadi, A.; Shi, J.; Lee, W.S.; Shi, P.; Kalam, A. Optimal PID-type fuzzy logic controller for a multi-input multi-output active magnetic bearing system. Neural Comput. Appl. 2016, 27, 2031-2046.

[26] Precup, R.-E.; David, R.-C.; Petriu, E.M.; Szedlak-Stinean, A.-I.; Bojan-Dragos, C.-A. Grey wolf optimizer-based approach to the tuning of Pl-fuzzy controllers with a reduced process parametric sensitivity. IFAC-Pap. Online 2016, 48, 55-60.

[27] Precup, R.-E.; David, R.-C.; Petriu, E.M. Grey wolf optimizer algorithm-based tuning of fuzzy control systems with reduced parametric sensitivity. IEEE Trans. Ind. Electron. 2017, 64, 527-534.

[28] S. Zhang, Y. Q, Zhou: Discr. Dyn. Nature Society. ID 481360 (2015), p. 1

[29] A. Zhu, C. Xu, Z. Li, J. Wu and Z. Liu: J. Syst. Eng. Elect. 26 (2015), p. 317
[30] SAREMI, S., S. MIRJALILI and S. M. MIRJALILI. Evolutionary Population Dynamics and Grey Wolf Optimizer. Neural Computing and Applications. 2015, vol. 26, iss. 5, pp. 1257-1263. ISSN 0941-0643.

[31] Singh, N. and Singh, S.B. "A Modified Mean Grey Wolf Optimization Approach for Benchmark and Biomedical Problems", Evolutionary Bioinformatics, 13(1), pp.1-28 (2017).

[32] Mittal, N. Singh, U. and Sohi, B.S. "Modified Grey Wolf Optimizer for Global Engineering Optimization", Applied Computational Intelligence and Soft Computing, 2016, Article id 7950348, pp. 1-16 (2016).

[33] Li, L. Sun, L. Guo, J. Qi, J. Xu, B. and Li, S. "Modified Discrete Grey Wolf Optimizer Algorithm for Multilevel Image Thresholding", Computational Intelligence and Neuroscience, 2017, Article id 3295769, pp. 1-16 (2017).

[34] Liu, H., Hua, G., Yin, H. and Xu, Y. "An Intelligent Grey Wolf Optimizer Algorithm for Distributed Compressed Sensing", Computational Intelligence Neuroscience, 2018, Article id 1723191, pp. 1-10 (2018).

[35] S.Mirjalili, s.M.mirjalili, and A.lewis,"grey wolf optimizer", advances in engineering software, vol.69, pp.46-61, 2014.

[36] Mech L D. Alphastatus,dominance, and division of labor in wolf packs. Can. J. Zool. Vol. 77, N¹, 1999. pp: 196-203.

[37] Muro C, Escobedo R, Spector L, Coppinger R. Wolf-pack (Canis lupus) hunting strategies emerge from simple rules in computational simulations. Behav Process 2011; 88:192-7.

[38] Mirjalili, S. Gandomi, A.H. Mirjalili, S.Z. Saremi, S., Faris, H. and Mirjalili, S.M." Salp Swarm Algorithm: A bio-inspired optimizer for engineering design problems", Advances in Engineering Software, vol. 114, pp. 163-191 (2017).

[39] MIRJALILI, S., S. SAREMI, S. M. MIRJALILI and L. S. COELHO. Multi-Objective Grey Wolf Optimizer: A Novel Algorithm for MultiCriterion Optimization. Expert Systems with Applications. 2016, vol. 47, iss. 1, pp. 106119. ISSN 0957-4174. DOI: 10.1016/j.eswa.2015.10.039.

[40] Emary, E.; Zawbaa, H.M.; Hassanien, A.E. Binary grey wolf optimization approaches for feature selection.Neurocomputing 2016, 172, 371-381.

[41] Jingwei Too, Abdul Rahim Abdullah, Norhashimah Mohd Saad, Nursabillilah Mohd Ali and Weihown Tee; "A New Competitive Binary Grey Wolf Optimizer to Solve the Feature Selection Problem in EMG Signals Classification", Computers 2018, 7, 58; doi:10.3390/computers7040058. 